\title{
Magnetoconductance of two point contacts in series
}

\author{
A. A. M. Staring, ${ }^{*}$ L. W. Molenkamp, and C. W. J. Beenakker \\ Philips Research Laboratories, NL-5600 JA Eindhoven, The Netherlands \\ L. P. Kouwenhoven \\ Delft University of Technology, NL-2600 GA Delft, The Netherlands \\ C. T. Foxon \\ Philips Research Laboratories, Redhill, Surrey RHI 5HA, England
}

(Received 6 November 1989)

\begin{abstract}
The magnetic-field dependence of the conductance of two opposite point contacts in series is studied experimentally, for magnetic fields extending into the quantum Hall effect regime. The magnetoconductance has a nonmonotonic, "camel-back" shape, in quantitative agreement with a recent theory. In addition, Aharonov-Bohm-type periodic magnetoconductance oscillations are observed, which are attributed to circulating edge states in a shallow potential basin between the point contacts.
\end{abstract}

The conductance of a constriction in a high-mobility two-dimensional electron gas (2D EG) measures directly the number of occupied one-dimensional subbands, $N$, in the constriction. This was demonstrated in the experiments by Van Wees et al. ${ }^{1}$ and Wharam et al. ${ }^{2}$ who discovered that the two-terminal conductance $G$ of such a quantum point contact is given approximately by

$$
G=\frac{2 e^{2}}{h} N
$$

A magnetic field $B$ perpendicular to the 2D EG depopulates the subbands, leading to a monotonic decrease ${ }^{3}$ of $G$ with $B$. The series conductance of two opposite point contacts in general does not have as simple a relation as Eq. (1) with the number of occupied subbands. In weak magnetic field the series conductance was found by Wharam et al. ${ }^{4}$ and Beton et al. ${ }^{5}$ to be strongly enhanced above the value which would follow from Ohmic addition of the separate point-contact resistances. As pointed out by Beenakker and Van Houten, ${ }^{6}$ this is due to the direct transmission of ballistic electrons from one point contact to the other, without intervening equilibration. The direct transmission probability is enhanced by the collimation of the electron beam injected into the 2D EG by a horn-shaped point contact. This horn collimation effect $^{6}$ was recently demonstrated experimentally. ${ }^{7}$

In stronger magnetic fields, where direct transmission is suppressed, a simple relation between the two-terminal series conductance $G_{\text {series }}$ and the number of occupied subbands becomes possible. This regime is the subject of the present paper. Theoretically, it was predicted in Ref. 6 that $G_{\text {series }}$ has a nonmonotonic ("camel-back" shaped) $B$ dependence, provided that the transmission from one point contact to the other occurs with intervening equilibration of the current-carrying edge states. This curious behavior results from the following relation between $G_{\text {series }}$ and the number of subbands: ${ }^{6}$

$$
G_{\text {series }}=\frac{2 e^{2}}{h}\left[\frac{1}{N_{1}}+\frac{1}{N_{2}}-\frac{1}{N_{L}}\right]^{-1} .
$$

Equation (2) results from the additivity of the fourterminal longitudinal magnetoresistance of the individual point contacts, ${ }^{6,8}$ which holds in the case of intervening equilibration. Here, $N_{1}$ and $N_{2}$ are the number of occupied subbands in the two point contacts, and $N_{L}$ is the number of Landau levels occupied in the region between the point contacts. At low magnetic fields $G_{\text {series }}$ first increases because the depopulation of the hybrid magnetoelectric subbands in the point contacts is delayed compared to the depopulation of Landau levels in the wide 2D EG between the point contacts. When the cyclotron radius becomes much smaller than the point-contact width, the confinement of the electrons in the point contacts is fully magnetic, and $G_{\text {series }}$ decreases as a result of the overall depopulation of Landau levels. Equation (2) is to be contrasted with the result which holds in the absence of equilibration, i.e., assuming adiabatic transport (a situation realized at moderate magnetic fields in the geometry of Ref. 9). One then has

$$
G_{\text {series }}=\frac{2 e^{2}}{h} \min \left\{N_{1}, N_{2}\right\},
$$

which, like the conductance of a single point contact, decreases monotonically with $B$. A nonmonotonic $B$ dependence is therefore a signature of equilibration among the edge states.

In this paper we present measurements of the magnetic-field dependence of the conductance of two opposite point contacts in series, for magnetic fields extending into the quantum-Hall-effect regime. We find good agreement with Eq. (2). In addition, we find an oscillatory structure superimposed on the magnetoconductance predicted by Eq. (2). This structure is periodic in the magnetic field and occurs only in transitions between the 
quantum Hall plateaus. We attribute it to an AharonovBohm-type quantum interference effect between edge states, similar to the effects observed in Refs. 10-12.

The measurements were performed on a sample consisting of two closely spaced opposite point contacts, defined in the 2D EG of a $\mathrm{GaAs}-\mathrm{Al}_{x} \mathrm{Ga}_{1-x}$ As heterojunction wafer by means of lateral depletion techniques. For sample fabrication we employ electron-beam lithography in a polymethylmethacrylate double-layer resist (using a Philips EBPG-4 Beamwriter) and lift off to deposit gold gates on top of a previously fabricated Hall-bar structure. The point contacts have a lithographic opening of $0.4 \mu \mathrm{m}$ and are separated by a $1-\mu \mathrm{m}$-wide, $18-\mu \mathrm{m}$ long channel (see inset in Fig. 1). The wide 2D EG has an electron density of $2.4 \times 10^{15} \mathrm{~m}^{-2}$ and a mobility of about $10^{2} \mathrm{~m}^{2} / \mathrm{V} \mathrm{s}$. The sample was kept at $100 \mathrm{mK}$ in a dilution refrigerator and the conductance measurements were performed using an ac lock-1n technique $(15 \mathrm{~Hz})$ with a small excitation voltage $(20 \mu \mathrm{V}$ over the sample and a series resistance of $3.3 \mathrm{k} \Omega$ ) in order to avold electron heating.

In Fig. 1 we show magnetoconductance curves obtained from a two-terminal measurement for the case of equal gate voltage $V_{g}$ on all gates defining the point contacts, which corresponds to $N_{1} \approx N_{2} \equiv N$. The observed $B$ dependence of $G_{\text {series }}$ can be described as a double peaked or "camel-back" shaped magnetoconductance (with peaks around $\pm 0.5 \mathrm{~T}$ ) with superimposed fine structure. The overall nonmonotonic $B$ dependence is as predicted by Eq. (2), and will be discussed in more detail below. Part of the fine structure consists of Shubnikov-de Haas ( $\mathrm{SdH}$ ) oscillations periodic in $1 / B$, noticeable as rapid oscillations around $\pm 0.5 \mathrm{~T}$, and broad dips in the quantum Hall plateaus at higher magnetic fields. These $\mathrm{SdH}$ oscillations originate in the wide $2 \mathrm{D}$

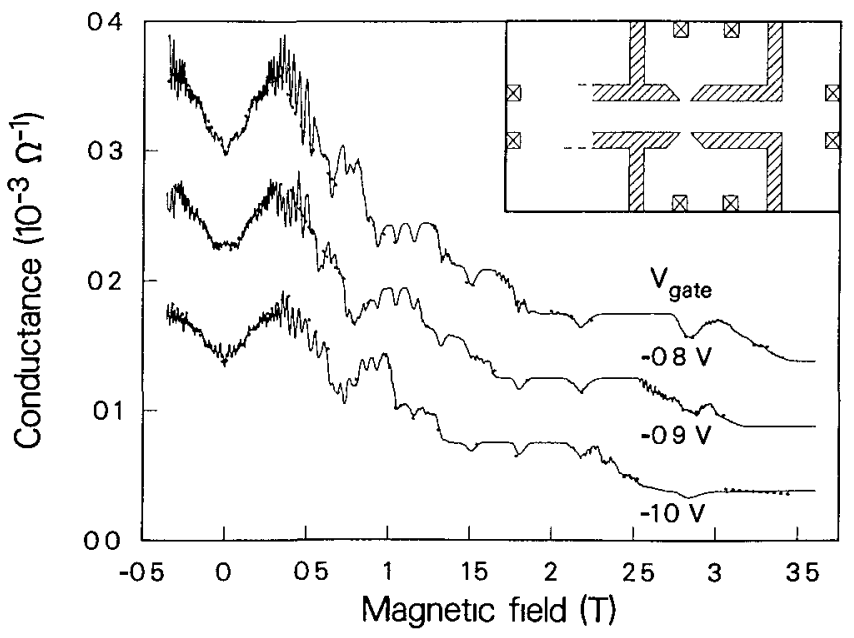

FIG 1. Two-terminal magnetoconductance measurements for three different values of the gate voltage (solid lines). For clarity, subsequent curves from bottom to top are offset by $05 \times 10^{-4} \Omega^{-1}$, with the $V_{g}=-1.0 \mathrm{~V}$ curve being shown at its actual value. The dotted lines are obtained by using Eqs (2), (5), and (6) with the values given in Table I. The inset gives a schematic diagram of the sample (not to scale) The pointcontact separation is $1 \mu \mathrm{m}$
EG leads, as can be deduced from their periodicity, which is a direct measure of the electron density. Around zero field reproducible rapid aperiodic oscillations are seen, reminiscent of the conductance fluctuations in disordered conductors. A more unusual fine structure occurs in the transitions between the quantum Hall plateaus, and consists of regular oscillations which are approximately perıdic in $B$. An enlargement of some of these oscillations is given in Fig. 2. We attribute these to the Aharonov-Bohm (AB) effect in a singly connected geometry. ${ }^{10-12}$

The proposed mechanism for these AB-type oscillations is 1llustrated in Fig. 3. Electrons in edge state (a) are transmitted without backscattering, and thus have transmission probability 1 . Those in edge state (b) can be transmitted via tunnelıng through the potential barrier in the point contacts to a circulating edge state (c), which follows the equipotentials at the guiding center energy $E_{G}=E_{F}-\left(n-\frac{1}{2}\right) \hbar \omega_{c}$ (with Landau-level index $n$ and cyclotron frequency $\omega_{c}$ ) in a shallow potential basin between the point contacts. The existence of such a poten-
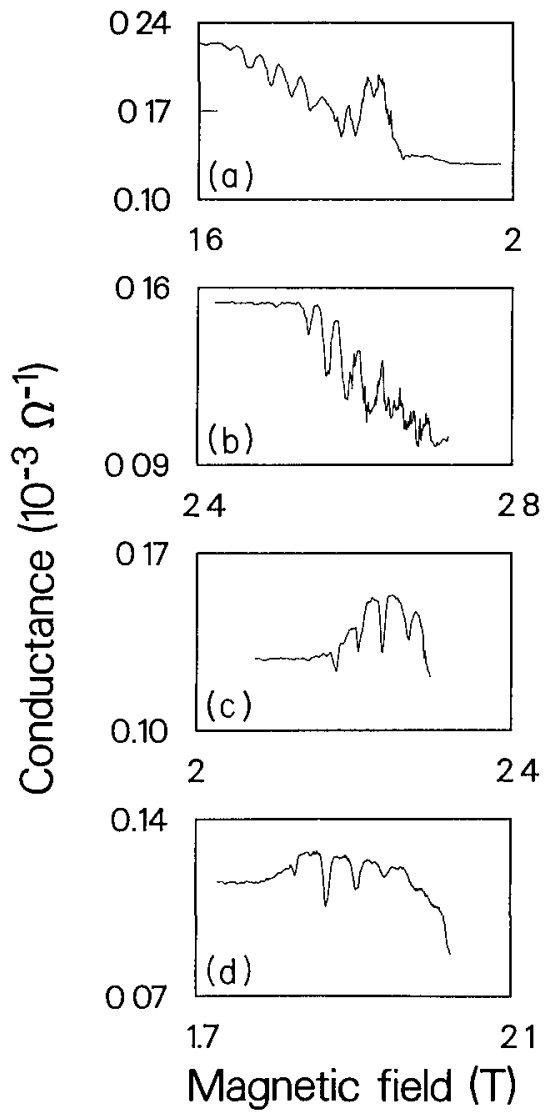

FIG. 2. Enlargement of the periodic oscrllations observed in the magnetoconductance for four different values of the gate voltage (a) $V_{g}=-08 \mathrm{~V}$, (b) $V_{g}=-09 \mathrm{~V}$, (c) $V_{g}=-10 \mathrm{~V}$, (d) $V_{g}=-1.1 \mathrm{~V}$ The $V_{\mathrm{g}}=-0.8 \mathrm{~V}$ oscillations are observed in the transition from quantum Hall plateau $l=3$ to $t=2$, the others in the $l=2$ to $l=1$ transition These measurements have been made in a four-terminal longitudinal configuration in order to suppress the Shubnikov-de Haas oscrllations, or iginating in the wide $2 \mathrm{D}$ EG leads 


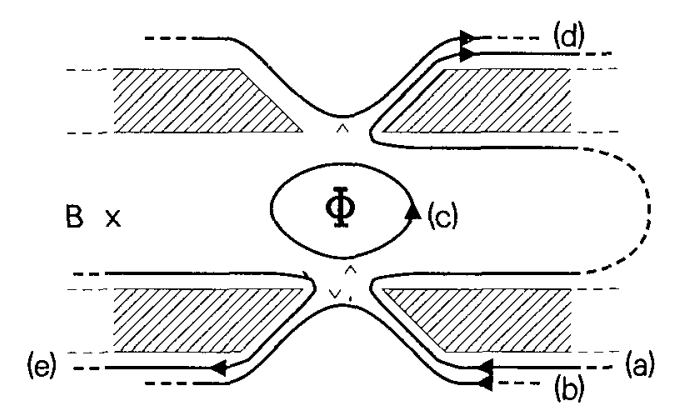

FIG. 3. Illustration of the proposed mechanism for the $A B$ like oscillations discussed in the text.

tial basin is in accord with a calculation of the electrostatics of a similar geometry by Kumar et al. ${ }^{13}$ The electrons can leave this circulating edge state by tunneling either back to edge state (b) or to edge state (d). An alternative to this mechanism of resonant transmission ${ }^{11}$ is resonant reflection ${ }^{14,15}$ from edge state (a) to (e) via the circulating edge state (c). This circulating edge state must enclose a flux $\Phi$ equal to an integer number of flux quanta $h / e$, i.e., $B A(B)=n h / e$, where $A(B)$ is the area enclosed by the circulating edge state (which depends on $B$ because of the $B$ dependence of the guiding center energy). This condition leads to a transmission and reflection probability which close to some field $B_{0}$ are periodic in $B$ with periodicity $\Delta B$ given by

$$
\begin{aligned}
\Delta B & =\frac{h}{e}\left[\left.\frac{d}{d B} B A(B)\right|_{B=B_{0}} ^{-1}\right. \\
& =\frac{h}{e}\left[A(B)+B \frac{d A}{d E_{G}} \frac{d E_{G}}{d B}\right]_{B=B_{0}}^{-1} .
\end{aligned}
$$

The second term in this expression for $\Delta B$ is negative since $d E_{G} / d B<0$ and $d A / d E_{G}>0$ for a typical potential basin, thus the effective area $A_{\text {eff }}$ calculated from the periodicity $\Delta B=h / e A_{\text {eff }}$ is a lower bound on the actual area $A$ of the basin. The periodicity in Fig. 2 varies between about 25 and $37 \mathrm{mT}$ (depending on gate voltage $V_{g}$ and magnetic field $B_{0}$ ), which corresponds to values of $A_{\text {eff }}$ between about $0.11 \times 10^{-12}$ and $0.16 \times 10^{-12} \mathrm{~m}^{2}$. A rough estimate of the basin area as the area of a circle of $1-\mu \mathrm{m}$ diameter (the point-contact separation) gives $A \approx 0.8 \times 10^{-12} \mathrm{~m}^{2}$, which is consistent with the lower bound given by $A_{\text {eff }}$, and forms presumably an estimate for the upper bound of the basin area.

In additional measurements of the magnetoconductance of a single point contact (not shown), performed on the same sample by grounding one pair of gates defining a point contact, the periodic oscillations in the transitions between the quantum hall plateaus, as well as the rapid aperiodic oscillations around zero field, are found to be absent. This demonstrates that this fine structure does not result from the background resistance or from an individual point contact, and is consistent with the mechanism proposed above.

We now turn to a quantitative analysis of the "camel- back" shaped magnetoconductance, in terms of Eq. (2), which is valid for magnetic fields such that direct transmission from one point contact to the other is suppressed. From measurements of the direct transmission probability, following the method of Ref. 7, we determine this to be the case for $|B|>0.1 \mathrm{~T}$. We assume a square-well confining potential in the point contacts (of width $W$ and electron density $n$ ), which should be a reasonable approximation at the low (negative) gate voltages considered. The number of occupied hybrid magnetoelectric subbands $N$ is given in this case by (assuming spin degeneracy and ignoring the discreteness of $N$ since we are interested in the overall behavior only $)^{3,16}$

$$
N= \begin{cases}\frac{h}{2 e} \frac{n}{B} \frac{2}{\pi}\left[\arcsin \xi+\xi\left(1-\xi^{2}\right)^{1 / 2}\right], & \text { if } \xi<1 \\ \frac{h}{2 e} \frac{n}{B}, & \text { if } \xi>1,\end{cases}
$$

where $\xi=W / 2 l_{\text {cycl }}$, and $l_{\text {cycl }}=\hbar(2 \pi n)^{1 / 2} / e B$ is the cyclotron radius inside the point contacts. The number of occupied Landau levels $N_{L}$ in the region of electron density $n_{s}$ between the point contacts is given by

$$
N_{L}=\frac{h}{2 e} \frac{n_{s}}{B} \text {. }
$$

In the following we shall assume that $n=n_{s}$, which we believe to be a reasonable approximation at the low (negative) gate voltages considered in Fig. 1 . The density in the region between the point contacts is reduced compared to the density of the wide 2D EG areas due to fringing fields from the gates, and is determined independently from the Hall effect (see Table I). The pointcontact width $W$ is treated as a free parameter in fits with the measured magnetoconductance.

The results of our analysis are given in Fig. 1 and Table I. The fits (dotted lines) were made using a constant background resistance, which is estimated at $2.0 \mathrm{k} \Omega$ (independent of the gate voltage) for low magnetic fields, from measurements of the quantized resistance of the individual point contacts as a function of the gate voltage. In principle, at higher magnetic fields a different value should be used because of the field dependence of the background resistance, which is expressed most clearly by the presence of Shubnikov-de Haas oscillations of the wide $2 \mathrm{D}$ EG regions. Because of the large resistance of the series point contacts at high magnetic fields, the fit is

TABLE I. Values used in Eqs. (2), (5), and (6) for obtaining the dotted lines in Fig. 1. The point-contact width $W$ is a fit parameter, the density $n$ in the narrow channel has been determined from the Hall effect, and the low-field background resistance $R_{b}$ is estimated as the difference between the resistance of an individual point contact and the quantized value given by Eq. (1).

\begin{tabular}{cccc}
\hline$V_{g}(\mathrm{~V})$ & $n\left(10^{15} \mathrm{~m}^{-2}\right)$ & $W(\mathrm{~nm})$ & $R_{g}(\mathrm{k} \Omega)$ \\
\hline-0.8 & 1.13 & 320 & 2.0 \\
-0.9 & 0.99 & 260 & 2.0 \\
-1.0 & 0.83 & 200 & 2.0 \\
\hline \hline
\end{tabular}


not very sensitive to these variations in the background resistance, and they are ignored for simplicity. Good agreement between Eq. (2) and the experimental data is obtained with $W$ as the only free parameter. Note that at $B=0$, Eq. (2) gives simple Ohmic addition of the pointcontact conductances, because direct transmission from one point contact to the other is neglected in that equation. Enhancement of the conductance above the value following from Ohmic addition is (for the measurements shown in Fig. 1) observed clearly at one of the gate voltages $\left(V_{g}=-0.9 \mathrm{~V}\right)$ only, but may be obscured at the other gate voltages by the aperiodic oscillations which are of the same order of magnitude. From the difference between Eq. (2) and the actual measurement (corrected for the background resistance) we find that $G_{\text {series }}$ is enhanced by $12 \%$ over $G / 2$, which is somewhat smaller than the enhancement of $20 \%$ which one would expect from measurements of the direct transmission probability. ${ }^{7}$ Clearly, the series resistance experiment is not an accurate way to determine the direct transmission probabil- ity.

We summarize our main results and conclusions. We have performed two-terminal magnetoconductance measurements of two opposite point contacts in series. The magnetoconductance shows the "camel-back" shape predicted previously, ${ }^{6}$ and is in quantitative agreement with the theory. In addition, an Aharonov-Bohm-type quantum interference effect is observed, which is attributed to resonant tunneling via circulating edge states in a shallow potential basin in the region between the point contacts.

The authors thank C. E. Timmering and M. E. I. Broekaart for sample fabrication, and R. Eppenga, H. van Houten, and J. G. Williamson for valuable discussions. One of us (A.A.M.S.) would like to thank Professor J. H. Wolter (Eindhoven University of Technology) and Professor M. F. H. Schuurmans (Philips Research Laboratories) for continuous encouragement and for providing the opportunity to carry out this work at the Philips Research Laboratories.
*Permanent address: Eindhoven University of Technology, $5600 \mathrm{MB}$ Eindhoven, The Netherlands.

${ }^{1}$ B. J. van Wees, H. van Houten, C. W. J. Beenakker, J. G. Williamson, L. P. Kouwenhoven, D. van der Marel, and C. T. Foxon, Phys. Rev. Lett. 60, 848 (1988).

${ }^{2}$ D. A. Wharam, T. J. Thornton, R. Newbury, M. Pepper, H. Ahmed, J. E. F. Frost, D. G. Hasko, D. C. Peacock, D. A. Ritchie, and G. A. C. Jones, J. Phys. C 21, L209 (1988).

${ }^{3}$ B. J. van Wees, L. P. Kouwenhoven, H. van Houten, C. W. J. Beenakker, J. E. Mooij, C. T. Foxon, and J. J. Harris, Phys. Rev. B 38, 3625 (1988).

${ }^{4}$ D. A. Wharam, M. Pepper, H. Ahmed, J. E. F. Frost, D. G. Hasko, D. C. Peacock, D. A. Ritchie, and G. A. C. Jones, J. Phys. C 21, L887 (1988).

${ }^{5}$ P. H. Beton, B. R. Snell, P. C. Main, A. Neves, J. R. OwersBradley, L. Eaves, M. Henini, O. H. Hughes, S. P. Beaumont, and C. D. W. Wilkinson, J. Phys. Condens. Matter 1, 7505 (1989).

${ }^{6}$ C. W. J. Beenakker and H. van Houten, Phys. Rev. B 39, 10445 (1989).

${ }^{7}$ L. W. Molenkamp, A. A. M. Staring, C. W. J. Beenakker, R. Eppenga, C. E. Timmering, J. G. Williamson, C. J. P. M. Harmans, and C. T. Foxon, Phys. Rev. B 41, 1274 (1990).

${ }^{8}$ H. van Houten, C. W. J. Beenakker, P. H. M. van Loosdrecht,
T. J. Thornton, H. Ahmed, M. Pepper, C. T. Foxon, and J. J. Harris, Phys. Rev. B 37, 8534 (1988).

${ }^{9}$ L. P. Kouwenhoven, B. J. van Wees, W. Kool, C. J. P. M. Harmans, A. A. M. Staring, and C. T. Foxon, Phys. Rev. B 40, 8083 (1989).

${ }^{10}$ P. H. M. van Loosdrecht, C. W. J. Beenakker, H. van Houten, J. G. Williamson, B. J. van Wees, J. E. Mooij, C. T. Foxon, and J. J. Harris, Phys. Rev. B 38, 10162 (1988).

${ }^{11}$ B. J. van Wees, L. P. Kouwenhoven, C. J. P. M. Harmans, J. G. Williamson, C. E. Timmering, M. E. I. Broekaart, C. T. Foxon, and J. J. Harris, Phys. Rev. Lett. 62, 2523 (1989).

${ }^{12}$ R. J. Brown, C. G. Smith, M. Pepper, M. J. Kelly, R. Newbury, H. Ahmed, D. G. Hasko, J. E. F. Frost, D. C. Peacock, D. A. Ritchie, and G. A. C. Jones, J. Phys. Condens. Matter 1, 6291 (1989).

${ }^{13}$ A. Kumar, S. E. Laux, and F. Stern, Bull. Am. Phys. Soc. 34, 589 (1989) and unpublished.

${ }^{14}$ J. K. Jain and S. A. Kivelson, Phys. Rev. Lett. 60, 1542 (1988).

${ }^{15}$ M. Büttiker, Phys. Rev. B 38, 12724 (1988).

${ }^{16}$ H. van Houten, C. W. J. Beenakker, J. G. Williamson, M. E. I. Broekaart, P. H. M. van Loosdrecht, B. J. van Wees, J. E. Mooij, C. T. Foxon, and J. J. Harris, Phys. Rev. B 39, 8556 (1989). 